\title{
Editorial
}

\section{Response to mutation and variants of the SARS-CoV-2 gene}

\author{
Edy Parwanto* \\ Department of Biology, Faculty of Medicine, Universitas Trisakti, Indonesia
}

Since the onset of the COVID-19 pandemic, our society has come to understand that the cause is the corona virus $(2019-\mathrm{nCoV}){ }^{(1)}$ or more popularly known as the severe acute respiratory syndrome coronavirus 2 (SARS-CoV-2). ${ }^{(2)}$ Further developments showed that SARS-CoV2 has mutated. It was reported in the UK on 18 December 2020 that SARS-CoV-2 gene mutations resulted in a new variant called B.1.1.7 or "variant under investigation, year 2020, month 12, variant 01" (VUI 202012/01). ${ }^{(3)}$ The name B.1.1.7 is derived from its phylogenetic heritage. Since the new viral variants are under study, they are also called "variant under investigation" or "variant of concern". ${ }^{(4)}$ For this reason, the SARS-CoV-2 lineage variant B.1.1.7 is also referred to as "variant of concern 202012/01" (VOC-202012/01). ${ }^{(5)}$ The B.1.1.7 variant of SARS-CoV-2 is also called 20I/501Y.V1.(6) Moreover, it has been demonstrated that line B.1.1.7 is associated with significant changes in the viral phenotype. (7)

The SARS-CoV-2 lineage variant B.1.1.7 is characterized by 8 gene mutations in the spike protein. For example, SARS-CoV-2 variant B.1.1.7 contains the mutations D614G and $\mathrm{N} 501 \mathrm{Y}$, that appear to increase the interaction between the spike protein and the angiotensin converting enzyme 2 (ACE2) receptor. ${ }^{(8)}$ Other researchers have also stated that the new variant of SARS-CoV-2 has an N501Y mutation in the spike protein. The new variant "may be associated" with the recent rise in cases in southeast England. Even so it is stated that the new variant of SARS-CoV-2 which is more contagious does not necessarily makes it more dangerous. It is further stated that a number of new variants have been detected in the UK, for example the D614G variant. In more detail, it is stated that the new variant VUI-202012/01 is defined by multiple spike protein mutations, including deletion 69-70, deletion 144, N501Y, A570D, D614G, P681H, T716I, S982A, D1118H. Subsequently it was reported that it was 14 days from $16^{\text {th }}$ December 2020 there has been a spike in COVID-19 cases in South East England, UK. ${ }^{(9)}$

Apart from type B.1.1.7 / 20I / 501Y.V1 of SARS-CoV-2 which appeared in the UK there is a new SARS-CoV-2 lineage 501Y.V2 which was found in Southern Africa and which emerged after the first epidemic wave in a severely affected metropolitan area, Nelson Mandela Bay, located on the coast of the Eastern Cape Province. This lineage spread rapidly, becoming within weeks the dominant lineage in the Eastern Cape and Western Cape Provinces. ${ }^{(10)}$ It was further stated that two of the substitutions in the 501Y.V2 lineage (E484K and N501Y) are within the receptor binding motif(RBM) associated with the function of binding with the human ACE2 (hACE2) receptor. Apart from the UK and South Africa, it has been reported that the B.1.1.7 variant of SARS-CoV-2 shows rapid transmission in the United States. ${ }^{(5)}$ Furthermore, a new variant of SARS-CoV-2, namely B.1.429 (also known as CAL.20C or 452R.V1), has been identified in California, USA. This strain is characterized by the presence of 5 mutations, namely ORF1a: I4205V, ORF1b: D1183Y, S: S13I; W152C; L452R. ${ }^{(11)}$

In response to the new variants of SARSCoV-2, various types of vaccines have been tested-At the community level, it is imperative that public health authorities communicate what the variants mean for people's day-to-day risk and explain why masking and physical distancing remain the best strategy for preventing disease 
and viral evolution. To overcome the new variant of SARS-CoV-2 which is based on the substitution of N501Y on the spike protein, isogenic N501 and Y 501 of SARS-CoV-2 have been made. The mRNA-based COVID-19 vaccine namely BNT162 b2 has been tested and has a neutralizing titer that is equivalent to the N501 and Y501 viruses. It shows the neutralizing effect of the BNT162b2 vaccine for mutant N501Y of SARSCoV-2. Neutralization of the SARS-CoV-2 N501Y mutant by BNT162b2 vaccine-elicited sera. ${ }^{(12)}$ Apart from these vaccines, it has been reported that the adenoviral vector vaccine namely ChAdOx1 nCoV-19 (AZD1222) is clinically effective in neutralizing the activity of the B.1.1.7 and non B.1.1.7 variants of SARS-CoV-2. Moreover, it was stated that the vaccine decreased the viral load of both B.1.1.7 and nonB.1.1.7 variants of SARS-CoV-2.(13)

As the world works to vaccinate, isolate, and treat communities to choke off the transmission, the virus is working to evade our scientific arsenal. Coordinated international efforts in genomic surveillance and phenotypic characterization of new strains will be critical if we hope to stay one step ahead of the virus.

\section{REFERENCES}

1. Zhou P, Yang X, Wang X, et al. A pneumonia outbreak associated with a new coronavirus of probable bat origin. Nature 2020;579:270-3. doi: 10.1038/s41586-020-2012-7.

2. World Health Organization. Naming the coronavirus disease (COVID-19) and the virus that causes it. Geneva: World Health Organization; 2020

3. Mahase E. Covid-19: what have we learnt about the new variant in the UK? BMJ 2020;371:m4944. http://dx.doi.org/10.1136/bmj.m4944.

4. Mascola JR, Graham BS, Anthony S, et al. SARSCoV-2 viral variants-tackling a moving target.
JAMA;2021:325:1261-2. doi: 10.1001/jama.2021. 2088.

5. Washington NL, Gangavarapu K, Zeller M, et al. Genomic epidemiology identifies emergence and rapid transmission of SARS-CoV-2 B.1.1.7 in the United States. medRxiv Preprint 2021. doi: 10.1101/ 2021.02.06.21251159.

6. Galloway SE, Paul P, MacCannell DR, et al. Emergence of SARS-CoV-2 B.1.1.7 lineage United States, December 29, 2020-January 12, 2021. Morb Mortal Wkly Rep 2021;70:95-9. DOI: http://dx.doi.org/10.15585/mmwr.mm7003e2.

7. Volz E, Mishra S, Meera CM, et al. Transmission of SARS-CoV-2 lineage B.1.1.7 in England: insights from linking epidemiological and genetic data. medRxiv Preprint 2021. doi : https://doi.org/ 10.1101/2020.12.30.20249034.

8. Villoutreix BO, Calvez V, Marcelin AG, Khatib AM. In silico investigation of the new UK (B.1.1.7) and South African (501Y.V2) SARS-CoV-2 variants with a focus at the ACE2-spike RBD interface. Int J Mol Sci 2021;22:1695. https:// doi.org/10.3390/ijms22041695.

9. European Centre for Disease Prevention and Control. Rapid increase of a SARS-CoV-2 variant with multiple spike protein mutations observed in the United Kingdom - 20 December 2020. ECDC: Stockholm; 2020.

10. Tegally H, Wilkinson E, Giovanetti M, et al. Detection of a SARS-CoV-2 variant of concern in South Africa. Nature 2021;592:438-43. https:// doi.org/10.1038/s41586-021-03402-9.

11. Zhang W, Davis BD, Chen SS, et al. Emergence of a novel SARS-CoV-2 strain in southern California, USA. medRxiv preprint 2021. doi: https://doi.org/10.1101/2021.01.18.21249786.

12. Xie X, LiuY, Liu J, et al. Neutralization of SARSCoV-2 spike 69/70 deletion, E484K and N501Y variants by BNT162b2 vaccine-elicited sera. Nat Med 2021;27: 620-1. https://doi.org/10.1038/ s41591-021-01270-4.

13. Moore JP. Approaches for optimal use of different COVID-19 vaccines: issues of viral variants and vaccine efficacy. JAMA 2021;325:1251-2. doi: 10.1001/jama.2021.3465. 